\title{
Outcome of Twin Pregnancies Complicated by a Neural Tube Defect
}

\author{
Vanessa Ross, ${ }^{1}$ Karen Reidy, ${ }^{2,3,4}$ Lex W. Doyle, 4,5,6,7 Ricardo Palma-Dias, ${ }^{1,2,3,4}$ and Mark P. Umstad ${ }^{1,4}$ \\ ${ }^{1}$ Division of Maternity Services, The Royal Women's Hospital, Melbourne, Victoria, Australia \\ ${ }^{2}$ Ultrasound Department, Pauline Gandel Imaging Centre, The Royal Women's Hospital, Melbourne, Victoria, Australia \\ ${ }^{3}$ Pregnancy Research Centre, The Royal Women's Hospital, Melbourne, Victoria, Australia \\ ${ }^{4}$ Department of Obstetrics and Gynaecology, University of Melbourne, Melbourne, Victoria, Australia \\ ${ }^{5}$ Department of Paediatrics, University of Melbourne, Melbourne, Victoria, Australia \\ ${ }^{6}$ Research Office, The Royal Women's Hospital, Melbourne, Victoria, Australia \\ ${ }^{7}$ Clinical Sciences, Murdoch Children's Research Centre, Melbourne, Victoria, Australia
}

\begin{abstract}
Twin pregnancies discordant for neural tube defects (NTD) is a management dilemma. Risks of preterm delivery from polyhydramnios must be balanced with the risks of selective termination (ST) of the anomalous fetus. We investigated the prevalence of twin pregnancies discordant for NTD and the rate of pregnancy complications in our institution over a 10-year period. Cases were obtained by searching the hospital ultrasound database and findings were confirmed by expert review of ultrasound images. Outcomes of ST and expectant management were assessed. Each unaffected co-twin was assigned to three consecutive twin pregnancy controls matched by chorionicity and maternal age. Primary outcome was birth before 34 weeks' gestation. Secondary outcomes were small for gestational age, mode of delivery, neonatal unit admission, and neonatal death. In total, 13 pregnancies were identified as potential cases. Of these, 11 were included in the analysis: 9 dichorionic diamniotic and 2 monochorionic diamniotic twins. Seven cases had ST and four were managed expectantly. We found 100\% (4/4) of expectantly managed pregnancies delivered $<34$ weeks compared with $14 \%(1 / 7)$ of the ST group $(p=.015)$. Polyhydramnios complicated three expectantly managed pregnancies and one pregnancy in the ST group. The birthweight SD score of all unaffected co-twins was $\geq-2$. The case-control analysis showed a higher rate of polyhydramnios in twin pregnancies discordant for NTD compared with controls, but little evidence for differences between groups in delivery rates $<34$ weeks, birthweight, neonatal unit admission, or neonatal death. ST warrants serious consideration to avoid potential complications to the unaffected co-twin.
\end{abstract}

Keywords: twins, neural tube defect, selective termination, expectant management, preterm birth

Twin pregnancies are at an increased risk of congenital anomalies compared with singleton pregnancies (Mastroiacovo et al., 1999; Windham \& Sever, 1982). In a majority of cases, the fetal anomaly is discordant and only one twin is affected (Bryan et al., 1987). This is also true of twin pregnancies complicated by neural tube defects (NTD). The prevalence of NTD in twins is $2.3 / 1,000$. These anomalies are seen in both monozygotic (MZ) and dizygotic (DZ) twin pairs, reflecting the multifactorial pattern of inheritance (Sebire et al., 1997).

Twin pregnancies discordant for NTD pose a management dilemma, as the unaffected co-twin is at risk of poor outcomes. Death of an affected twin in utero can have devastating effects on the unaffected co-twin, particularly in monochorionic (MC) pregnancies where the unaffected twin has an increased risk of both neurological damage and death (Fusi \& Gordon, 1990). In studies involving anencephaly, rates of fetal death in utero (FDIU) of the affected twin have been reported to be approximately $25 \%$, which is accompanied by the death of the unaffected cotwin in the majority of cases (Sebire et al., 1997). In addition, the development of polyhydramnios complicates 36$57 \%$ of cases (Lipitz et al., 1995; Vandecruys et al., 2006).

RECEIVED 30 September 2017; ACCEPTED 9 April 2018. First published online 7 May 2018.

ADDRESS FOR CORRESPONDENCE: Mark Umstad, Division of Maternity Services, The Royal Women's Hospital, 20 Flemington Road, Parkville, Victoria 3052, Australia. E-mail: Mark.Umstad@thewomens.org.au 
Expectant management of polyhydramnios has been associated with preterm delivery rates of up to $100 \%$ (Leeker \& Beinder, 2004), exposing the unaffected co-twin to higher rates of perinatal morbidity and mortality (Nassar et al., 2000). Selective termination (ST) of the affected twin is an option offered to parents to minimize risks to the pregnancy and the unaffected co-twin. The technique used is determined by chorionicity. Intracardiac potassium chloride or lignocaine injection is preferred for dichorionic (DC) twins, while cord occlusion methods are preferred for MC twins. ST carries a risk of pregnancy loss that is dependent on the gestation when the procedure is performed, the technique used, and the experience of the operator (Evans et al., 1994; Lipitz et al., 1995).

In DC twins, ST is associated with a cumulative loss rate of 5\% (Bigelow et al., 2014). The gestational age at the time of ST is associated with both pregnancy loss rates and preterm delivery rates (Dural et al., 2017), and it is recommended the procedure is performed prior to 18 weeks' gestation (Bigelow et al., 2014).

In MC twins, the risk of pregnancy loss following cord occlusion techniques is higher and the procedures are rarely performed prior to the second trimester. A recent systematic review and meta-analysis by Gaerty et al. (2015) has analyzed the perinatal outcomes of MC pregnancies who undergo either radiofrequency ablation or bipolar cord occlusion for ST, and found that neither method was superior to the other. It has also been noted that ST of the presenting MC twin has a higher rate of delivery $<34$ weeks' gestation, regardless of the method used (Yinon et al., 2015).

To date, there are no large series assessing twin pregnancies discordant for NTD. The current study aimed, first, to compare two different management options (ST vs. expectant management) in twin pregnancies discordant for NTD and the associated pregnancy and neonatal outcomes; and, second, to contrast outcomes between twin pregnancies discordant for NTD (cases) and twin pregnancies with no NTD (controls) to determine the overall burden of twin pregnancies with discordant NTD relative to twin pregnancies with no NTD.

\section{Patients and Methods}

This is a retrospective cohort study of all twin pregnancies affected by a NTD at the Royal Women's Hospital, Melbourne (RWH) over a 10-year period. Cases were obtained from a search of the hospital's ultrasound Picture Archiving and Communication System (PACS) database. All the cases of multiple pregnancy discordant for a NTD were included. Each case was reviewed by an experienced obstetrician with ultrasound expertise to confirm the findings.

Monochorionic diamniotic (MCDA) and dichorionic diamniotic (DCDA) cases only were included. Monochorionic monoamniotic (MCMA) cases were not included as outcomes for these women and their fetuses are complex and largely determined by the risks of monoamnionicity (Shub \& Walker, 2015). Each eligible case was matched with three controls who were selected chronologically from the three subsequent multiple pregnancy ultrasounds with the same chorionicity and maternal age (within 5 years). Multiple pregnancies with other major fetal anomalies were excluded from the control group. In addition, the controls were required to have a normal first trimester ultrasound scan result (between 11 weeks and 13.9 weeks). Controls found to have any fetal anomaly or a nuchal translucency (NT) above the 99th percentile $(>3.5 \mathrm{~mm})$ were excluded. Further control exclusion criteria included women with significant chronic medical conditions, such as diabetes or hypertension, or those in whom the pregnancy outcome data were unavailable for review. Background and outcome data were collected for the mother and both twins for cases and controls.

The primary outcome was birth before 34 weeks' gestation. The secondary outcomes were small for gestational age (birthweight $<-2 S D$ relative to Australian twin birthweight percentiles; Li et al., 2015), mode of delivery, admission to a neonatal unit (either the neonatal intensive care unit or the specialcare nursery), or neonatal death.

Data were analyzed using Stata version 14.2. First, we were interested in outcomes confined within the twin pregnancies discordant for NTD between those treated with ST and those treated expectantly where differences between groups were contrasted by chi-square (or Fisher's exact test), or $t$ tests (or Mann-Whitney U Test), as appropriate. Second, twin pregnancies discordant for NTD were compared with those with no NTD, using generalized estimating equations (GEEs) with robust (sandwich) estimates of standard errors to allow for clustering of the three controls for each case. Mean differences with 95\% confidence intervals (CIs) or odds ratios (ORs) were calculated from the regression coefficients and their standard errors. For neonatal outcomes, infant characteristics were compared between non-affected cases and their corresponding controls, matched for birth order.

Human Research Ethics Committee (HREC) approval was obtained for a retrospective anonymized audit project that met the criteria for quality assurance activities outlined in the National Health and Medical Research Council guideline 'Ethical Considerations in Quality Assurance and Evaluation Activities' (National Health \& Medical Research Council, 2014).

\section{Results}

Between 2004 and 2014, there were over 1,800 twin pregnancies managed at our hospital. Thirteen were complicated by a discordant NTD: eight with anencephaly, three with an encephalocoele, and two with spina bifida. Nine of the 13 pregnancies were DCDA, three were MCDA, and one was MCMA. The mean gestation at diagnosis was 


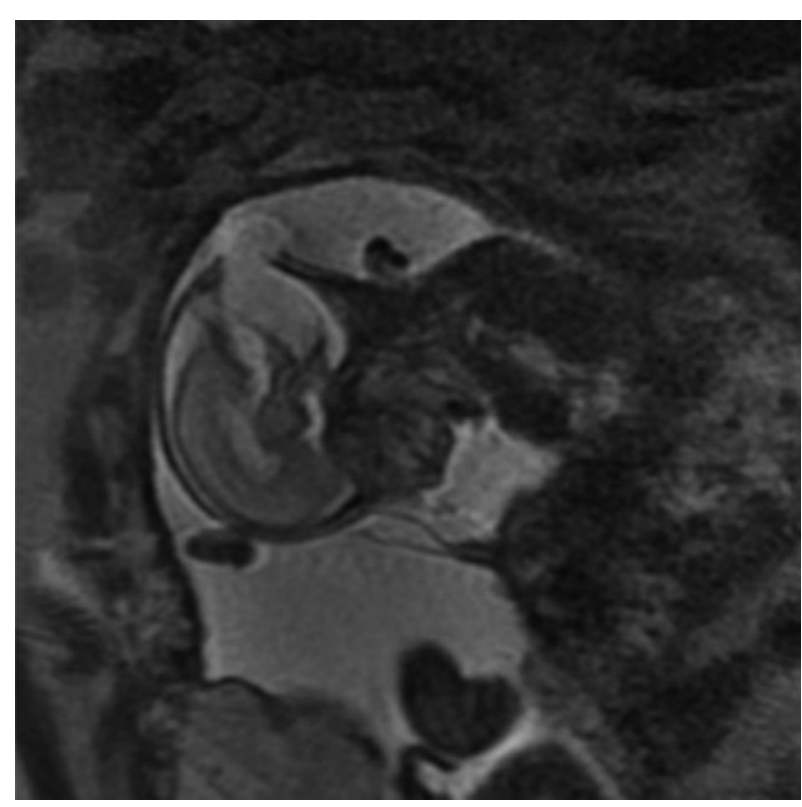

\section{FIGURE 1}

MRI of twin with encephalocoele.

13.7 weeks ( $S D$ 1.0) for anencephaly, 18.1 weeks ( $S D 0.4$ ) for spina bifida, and 20.4 weeks (SD 3.2) for encephalocoele. Figure 1 shows an ultrasound image of a twin pregnancy discordant for encephalocoele. Among cases with a NTD, six affected fetuses and three unaffected co-twins had diagnostic genetic testing and all were found to have a normal karyotype.

The MCMA pregnancy had ST for anencephaly at 15.1 weeks and subsequently underwent a misoprostol induction following demise of the co-twin at 15.9 weeks; this case was excluded from further analysis. In one MCDA twin pregnancy, parents elected to have a termination of the whole pregnancy at 16 weeks' gestation; this case was also excluded.

\section{Pregnancies Discordant for NTD}

Of the remaining 11 affected twin pregnancies, the affected fetus had ST in seven cases (64\%), and four were managed expectantly (36\%). The seven cases in which ST was performed comprised three for anencephaly, two for encephalocoele, and two for spina bifida. One of the seven cases delivered at 19 weeks following ST of the affected presenting anencephalic twin at 14 weeks' gestation; although liveborn, the infant died quickly after birth. The remaining six pregnancies where ST was performed resulted in a live term ( $\geq 37$ weeks' gestation) delivery.

The four cases managed expectantly included three pregnancies with an anencephalic fetus and one with an encephalocoele. Two women in this expectant management group, both DCDA pregnancies, required tocolysis for preterm labor at 28 weeks' and 29 weeks' gestation, re- spectively. The first woman delivered at 29 weeks' gestation and the unaffected co-twin was diagnosed with a right grade 1 intraventricular hemorrhage at 7 days of age. The baby was also treated for hyaline membrane disease, a right pneumothorax, and jaundice and was discharged home at 53 days of age. The second woman developed pre-eclampsia and delivered at 30 weeks' gestation. The unaffected co-twin was diagnosed with a large patent ductus arteriosus, which was treated with indomethacin. The baby also had hyaline membrane disease and jaundice and was discharged home at 46 days of age.

The two remaining MCDA pregnancies managed expectantly delivered at 31 weeks' and 32 weeks' gestation, respectively, and both unaffected co-twins had adverse outcomes. One co-twin was diagnosed with sepsis and widespread intraventricular hemorrhage, resulting in a neonatal death at 10 days of age. The other co-twin was diagnosed with periventricular leukomalacia following birth.

Polyhydramnios complicated one (14\%) case following ST and three $(75 \%)$ cases in the expectant management group (OR 0.06; 95\% CI [0.003, 1.23]; Fisher's exact test; $p=.09)$.

The mean gestation at delivery in the ST group was 35.5 weeks (SD 7.1) compared with 31.0 weeks (SD 1.5) in the expectant management group, a non-significant difference (mean difference 4.5 weeks; 95\% CI [-3.7, 12.9 weeks], $p=$ $.25)$. Rates of delivery $<34$ weeks' gestation were $14 \%(1 / 7)$ of those who underwent ST compared with $100 \%(4 / 4)$ of expectantly managed pregnancies (Fisher's exact test; $p=.015)$.

Delivery was by cesarean section in 8 of the 11 cases. Four unaffected twins required admission to the neonatal unit, primarily due to prematurity; all were in the expectant management group (Fisher's exact test; $p=.003$ ). Two liveborn infants with anencephaly had birthweight $S D$ scores $<-2$, whereas the birthweight $S D$ scores of all unaffected cotwins were $\geq-2$.

\section{Case-Control Analysis}

When comparing outcomes of the cases (discordant for NTD) with the controls (not discordant for NTD), there were few differences in demographic or antenatal variables, although more of the cases were smokers and more had polyhydramnios (Table 1). The mode of delivery was similar between the groups with a majority of women undergoing a cesarean section.

The infant outcomes comparing the unaffected case twins with their matched controls are shown in Table 2.

There were no substantial differences in sex, mean gestational age, birthweight, or birthweight $S D$ score between the groups; no twins in either group had birthweight $S D$ scores $<-2$. Although more cases delivered $<34$ weeks than controls, the evidence for a difference was weak after accounting for the lack of independence between controls in the analyses. The groups had similar rates of admission 
TABLE 1

Demographic and Pregnancy Data for Cases and Controls

\begin{tabular}{|c|c|c|c|c|}
\hline Variable & Cases $(n=11)$ & Controls $(n=33)$ & Statistics & \\
\hline Maternal age: mean (SD) & $32.6(6.4)$ & $32.7(5.7)$ & $-0.1[-0.9,0.8]^{\dagger}$ & $p=.89$ \\
\hline Smoking: $n(\%)$ & $3 / 9(33)$ & $2 / 30(7)$ & $5.86[1.04,32.5]^{\ddagger}$ & $p=.045$ \\
\hline Parity & $1(0,1)$ & $0(0,1)$ & $z=1.43^{*}$ & $p=.23^{*}$ \\
\hline ART: $n(\%)$ & $2 / 10(20)$ & $17(52)$ & $0.25[0.06,1.09]^{\ddagger}$ & $p=.06$ \\
\hline Chorionicity DCDA: $n(\%)$ & $9(82)$ & $27(82)$ & NA & \\
\hline Chorionicity MCDA: $n(\%)$ & $2(18)$ & $6(18)$ & NA & \\
\hline Antenatal corticosteroids: $n(\%)$ & $6(55)$ & $14(42)$ & $1.63[0.35,7.48]^{\ddagger}$ & $p=.53$ \\
\hline Chorioamnionitis: $n(\%)$ & $2(18)$ & $2(6)$ & $3.44[0.33,36.3]^{\ddagger}$ & $p=.30$ \\
\hline TTTS: $n(\%)$ & $0(0)$ & $0(0)$ & NA & \\
\hline Polyhydramnios: $n(\%)$ & $4(36)$ & $1(3)$ & $18.3[1.30,257]^{\ddagger}$ & $p=.031$ \\
\hline Cesarean delivery: $n(\%)$ & $8(73)$ & $21(64)$ & $1.52[0.59,3.95]^{\ddagger}$ & $p=.39$ \\
\hline \multicolumn{5}{|c|}{ 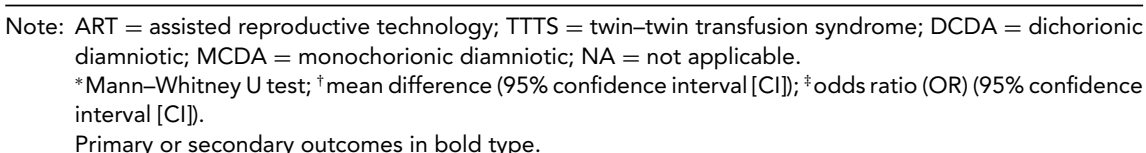 } \\
\hline
\end{tabular}

TABLE 2

Infant Data for Non-affected Cases and Matched Control Twins

\begin{tabular}{|c|c|c|c|c|}
\hline Variable & Cases $(n=11)$ & Controls $(n=33)$ & Statistics & \\
\hline Male: $n(\%)$ & $5(45)$ & $20(61)$ & $0.54[0.13,2.20]$ & $p=.39$ \\
\hline Gestational age at birth $(w)$ : mean $(S D)$ & $\begin{array}{l}33.9(6.0) \\
37.1(30.1,38.7)^{\dagger}\end{array}$ & $\begin{array}{l}35.8(3.6) \\
37.0(34.6,37.4)^{\dagger}\end{array}$ & $-1.9[-5.7,1.95]^{\S}$ & $p=.32$ \\
\hline Delivery <34 weeks: $n(\%)$ & $5(45)$ & 4 (12) & $6.04[0.85,42.7] \rrbracket$ & $p=.071$ \\
\hline Liveborn: $n(\%)$ & $11(100)$ & $32(97)$ & NA & \\
\hline Birthweight (g): mean (SD) & $\begin{array}{l}2,384(1,198) \\
2,930(1,376,3,210)^{\dagger}\end{array}$ & $\begin{array}{l}2,502(645) \\
2,645(2,218,2,913)^{\dagger}\end{array}$ & $-1,185[-836,598]^{\S}$ & $p=.75$ \\
\hline Birthweight SD score: mean (SD) & $0.94(1.16) n=10$ & $0.20(0.86) n=32$ & $0.75[-0.16,1.66]^{\S}$ & $p=.10$ \\
\hline Birthweight $<-2$ SD: $n(\%)$ & $0(0)$ & $0(0)$ & NA & \\
\hline Apgar score at 5 minutes & $9(8,9)$ & $9(9,9)$ & $z=0.10$ & $p=.92^{*}$ \\
\hline NICU admission: $n(\%)$ & $4(36)$ & $4 / 32(12)$ & $4.08[0.71,23.3]$ & $p=.11$ \\
\hline SCN admission: $n(\%)$ & $4(36)$ & $15 / 32(47)$ & $0.65[0.17,2.47]$ & $p=.53$ \\
\hline Respiratory distress: $n(\%)$ & $3(27)^{\ddagger}$ & $6(19)$ & $1.64[0.37,7.19]$ & $p=.51$ \\
\hline Nasal CPAP only: $n$ (\%) & $3(27)$ & $4 / 32(12)$ & $2.66[0.47,15.0]$ & $p=.27$ \\
\hline Breast feeding at discharge: $n(\%)$ & $7 / 10(70)$ & $25 / 32(78)$ & $0.66[0.15,3.01]$ & $p=.60$ \\
\hline NND: $n(\%)$ & $2(18)$ & $0(0)$ & NA & \\
\hline
\end{tabular}

Note: $\mathrm{w}=$ weeks; $\mathrm{SD}=$ standard deviation; $\mathrm{NICU}=$ neonatal intensive care unit; $\mathrm{SCN}=$ special care nursery; NND = neonatal death $\mathrm{NA}=$ not applicable.

${ }^{*}$ Mann-Whitney $U$ test; ${ }^{\dagger}$ median (25th-75th centiles); ${ }^{*} n=2$ intubated and treated with exogenous surfactant; ${ }^{\S}$ mean difference (95\% Cl); odds ratio (OR) (95\% confidence interval [Cl])

Primary or secondary outcomes in bold type.

to the neonatal intensive care unit or special care nursery, respiratory distress, and use of respiratory support, and most in both groups were being breastfed on discharge home. The indication for admission to the nurseries in the majority of infants was for prematurity; however, one term infant was admitted with hypoglycaemia at 37 weeks' gestation. The only two neonatal deaths occurred among the cases, as described above.

\section{Discussion}

The main finding in our study comparing management options within twins discordant for NTD was that expectant management resulted in more births $<34$ weeks, with consequently more infants requiring admission to the nursery than did ST. The main finding from the case-control analysis was that twin pregnancies discordant for NTD were more likely to develop polyhydramnios, with a trend for more to deliver $<34$ weeks than twin pregnancies with no NTD.
It has long been recognized that twin pregnancies with a single anomalous fetus have poorer outcomes compared with normal twin pregnancies (Sun et al., 2009). The optimal management of twin pregnancies discordant for NTD is complicated due to the scarcity of evidence in the literature and small sample sizes, and hence a lack of power to detect important differences between groups. A limitation of our study was that it is also small; however, ST resulted in some clinical benefits compared with expectant management. Currently, parents have the option of terminating the entire pregnancy, ST of the anomalous fetus, or expectant management involving monitoring for polyhydramnios and consideration of amniodrainage if required.

ST has previously been shown to prolong gestation at delivery for the unaffected co-twin and this directly correlates with a higher birthweight and better perinatal outcomes (Gul et al., 2005). It does, however, carry a risk of pregnancy loss of up to 5\% in DC twins (Bigelow et al., 2014) and up to $11 \%$ in MC twins (Gaerty et al., 2015), consistent 
with the rate of neonatal deaths in the unaffected co-twin in our study. The risk of FDIU of the unaffected co-twin is reported to be greatest in the two weeks post-procedure (Rossi \& D'Addario, 2009). These findings must be taken into consideration when counselling parents about management options. ST in MC twins is a viable option and a meta-analysis by Rossi and D'Addario (2009) showed that outcomes were better for MC twins undergoing ST after 18 weeks, which is contrary to findings for ST in DC twins. Unfortunately, ST does not reduce perinatal mortality overall (Lust et al., 2008).

Considering the risks associated with ST (including miscarriage, infection, and prelabor preterm rupture of the membranes), as well as the findings of some studies that there is no increased risk of preterm delivery in twin pregnancies discordant for fetal anomalies (Chang et al., 2004; Linskens et al., 2011), it is important to consider expectant management options. Close antenatal monitoring with serial ultrasound examinations for the early diagnosis of polyhydramnios is one option. Although some will develop polyhydramnios, approximately $65 \%$ of these cases can be managed expectantly, and amniodrainage or ST can be considered for the remainder of cases (Vandecruys et al., 2006).

When comparing the rates of delivery $<34$ weeks for the NTD cases (independent of management type) and the control group, $45 \%$ of all cases were delivered $<34$ weeks' gestation compared with only $12 \%$ of controls, but the small sample size reduced the strength of the evidence for a difference between groups. The preterm birth rates in our study overall reflect the known higher rates of preterm delivery seen with normal twin gestations (Goldenberg et al., 1996; Petit et al., 2011).

With respect to neonatal outcomes, the unaffected cotwin is no more likely to be small for gestational age or require admission to NICU or SCN than matched controls. MC pregnancies have added complexities for management, and it is well described that neurological injury and preterm delivery in MC twins are the most common cause of neonatal morbidity. Death of an affected twin in utero also places the unaffected co-twin at a significant risk (Evans et al., 1994). Both of the MC co-twins in our study suffered poor neonatal outcomes.

Cesarean delivery rates were no different for cases and controls, and this supports the findings of Nassar et al. (2000), who assessed discordant major structural anomalies in twin pregnancies, including NTD. Interestingly, half of the affected twin pregnancies undergoing cesarean section for delivery was for the indication of malpresentation; however, this was likely gestation dependent.

The current study offers insight into the management dilemmas seen with twin pregnancies complicated by NTD. The main strength of the current study is that it is the first case-control analysis of twin pregnancies discordant for an NTD. The major limitation was the small number of cases available for analysis due to the rarity of the condition. The current study may therefore be underpowered to detect clinically important differences. Firm recommendations for management cannot be made without larger randomized controlled trials comparing management options.

\section{Conclusions}

Our study has confirmed that polyhydramnios and preterm delivery may complicate twin pregnancies affected with an NTD, but they are not universal. While ST is not without risk, it warrants serious consideration to avoid the potential complications to the unaffected co-twin.

\section{Acknowledgments}

This work was supported by the Operational Infrastructure Support Program of the State Government of Victoria.

\section{Disclosure of Interests}

None of the authors have any conflicts of interest to report.

\section{References}

Bigelow, C. A., Factor, S. H., Moshier, E., Bianco, A., Eddleman, K. A., \& Stone, J. L. (2014). Timing of and outcomes after selective termination of anomalous fetuses in dichorionic twin pregnancies. Prenatal Diagnosis, 34, 1320-1325.

Bryan, E., Little, J., \& Burn, J. (1987). Congenital anomalies in twins. Baillière's Clinical Obstetrics and Gynaecology, 1, 697-721.

Chang, Y. L., Chao, A. S., Cheng, P. J., Chung, C. L., Chueh, H. Y., Chang, S. D., \& Soong, Y. K. (2004). Presence of a single fetal major anomaly in a twin pregnancy does not increase the preterm rate. Australian and New Zealand Journal of Obstetrics and Gynaecology, 44, 332-336.

Dural, O., Yasa, C., Kalelioglu, I. H., Can, S., Yilmaz, G., Corbacioglu Esmer, A., ... Yuksel, A. (2017). Comparison of perinatal outcomes of selective termination in dichorionic twin pregnancies performed at different gestational ages. Journal of Maternal-Fetal \& Neonatal Medicine, 30, 1388-1392.

Evans, M. I., Goldberg, J. D., Dommergues, M., Wapner, R. J., Lynch, L., Dock, B. S., ... Berkowitz, R. L. (1994). Efficacy of second-trimester selective termination for fetal abnormalities: International collaborative experience among the world's largest centers. American Journal of Obstetrics and Gynecology, 171, 90-94.

Fusi, L., \& Gordon, H. (1990). Twin pregnancy complicated by single intrauterine death. Problems and outcome with conservative management. British Journal of Obstetrics and Gynaecology, 97, 511-516.

Gaerty, K., Greer, R. M., \& Kumar, S. (2015). Systematic review and metaanalysis of perinatal outcomes after radiofrequency ablation and bipolar cord occlusion in monochorionic pregnancies. American Journal of Obstetrics and Gynecology, 213, 637-643. 
Goldenberg, R. L., Iams, J. D., Miodovnik, M., Van Dorsten, J. P., Thurnau, G., Bottoms, S., ... McNellis, D. (1996). The preterm prediction study: Risk factors in twin gestations. National Institute of Child Health and Human Development Maternal-Fetal Medicine Units Network. American Journal of Obstetrics and Gynecology, 175, 1047-1053.

Gul, A., Cebeci, A., Aslan, H., Polat, I., Sozen, I., \& Ceylan, Y. (2005). Perinatal outcomes of twin pregnancies discordant for major fetal anomalies. Fetal Diagnosis and Therapy, 20, 244-248.

Leeker, M., \& Beinder, E. (2004). Twin pregnancies discordant for anencephaly-management, pregnancy outcome and review of literature. European Journal of Obstetrics \& Gynecology and Reproductive Biology, 114, 15-18.

Li, Z., Umstad, M. P., Hilder, L., Xu, F., \& Sullivan, E. A. (2015). Australian national birthweight percentiles by sex and gestational age for twins, 2001-2010. BMC Pediatrics, 15, 148.

Linskens, I. H., Elburg, R. M., Oepkes, D., Vugt, J. M., \& Haak, M. C. (2011). Expectant management in twin pregnancies with discordant structural fetal anomalies. Twin Research and Human Genetics, 14, 283-289.

Lipitz, S., Meizner, I., Yagel, S., Shapiro, I., Achiron, R., \& Schiff, E. (1995). Expectant management of twin pregnancies discordant for anencephaly. Obstetrics \& Gynecology, 86, 969-972.

Lust, A., De Catte, L., Lewi, L., Deprest, J., Loquet, P., \& Devlieger, R. (2008). Monochorionic and dichorionic twin pregnancies discordant for fetal anencephaly: A systematic review of prenatal management options. Prenatal Diagnosis, 28, 275-279.

Mastroiacovo, P., Castilla, E. E., Arpino, C., Botting, B., Cocchi, G., Goujard, J., ... Rosano, A. (1999). Congenital malformations in twins: An international study. American Journal of Medical Genetics, 83, 117-124.

Nassar, A. H., Adra, A. M., Gomez-Marin, O., \& O’Sullivan, M. J. (2000). Perinatal outcome of twin pregnancies with one structurally affected fetus: A case-control study. Journal of Perinatology, 20, 82-86.

National Health \& Medical Research Council (NHMRC). (2014). Ethical considerations in quality assurance and evaluation activities. Canberra, ACT Australia: Author. Retrieved from https://www.nhmrc.gov.au/_files_nhmrc/ publications/attachments/e111_ethical_considerations_ in_quality_assurance_140326.pdf.

Petit, N., Cammu, H., Martens, G., \& Papiernik, E. (2011). Perinatal outcome of twins compared to singletons of the same gestational age: A case-control study. Twin Research and Human Genetics, 14, 88-93.

Rossi, A. C., \& D’Addario, V. (2009). Umbilical cord occlusion for selective feticide in complicated monochorionic twins: A systematic review of literature. American Journal of Obstetrics and Gynecology, 200, 123-129.

Sebire, N. J., Sepulveda, W., Hughes, K. S., Noble, P., \& Nicolaides, K. H. (1997). Management of twin pregnancies discordant for anencephaly. British Journal of Obstetrics and Gynaecology, 104, 216-219.

Shub, A., \& Walker, S. P. (2015). Planned early delivery versus expectant management for monoamniotic twins. Cochrane Database of Systematic Reviews, 2015(4), CD008820. doi:10.1002/14651858.CD008820.pub2

Sun, L. M., Chen, X. K., Wen, S. W., Fung, K. F., Yang, Q., \& Walker, M. C. (2009). Perinatal outcomes of normal cotwins in twin pregnancies with one structurally anomalous fetus: A population-based retrospective study. American Journal of Perinatology, 26, 51-56.

Vandecruys, H., Avgidou, K., Surerus, E., Flack, N., \& Nicolaides, K. H. (2006). Dilemmas in the management of twins discordant for anencephaly diagnosed at $11+0$ to 13 +6 weeks of gestation. Ultrasound in Obstetrics \& Gynecology, 28, 653-658.

Windham, G. C., \& Sever, L. E. (1982). Neural tube defects among twin births. American Society of Human Genetics, 34, 988-998.

Yinon, Y., Ashwal, E., Weisz, B., Chayen, B., Schiff, E., \& Lipitz, S. (2015). Selective reduction in complicated monochorionic twins: Prediction of obstetric outcome and comparison of techniques. Ultrasound in Obstetrics \& Gynecology, $46,670-677$. 